\title{
Incremental Hashing for SPIN
}

\author{
Viet Yen Nguyen ${ }^{1}$ and Theo C. Ruys ${ }^{2}$ \\ 1 RWTH Aachen University, Germany. \\ http://www-i2.cs.rwth-aachen.de/ nguyen/ \\ ${ }^{2}$ University of Twente, The Netherlands. \\ http://www.cs.utwente.nl/ ruys/
}

\begin{abstract}
This paper discusses a generalised incremental hashing scheme for explicit state model checkers. The hashing scheme has been implemented into the model checker SPIN. The incremental hashing scheme works for SPIN's exhaustive and both approximate verification modes: bitstate hashing and hash compaction. An implementation has been provided for 32-bit and 64-bit architectures.

We performed extensive experiments on the BEEM benchmarks to compare the incremental hash functions against SPIN's traditional hash functions. In almost all cases, incremental hashing is faster than traditional hashing. The amount of performance gain depends on several factors, though.

We conclude that incremental hashing performs best for the (64-bits) SPIN's bitstate hashing mode, on models with large state vectors, and using a verifier, that has been optimised by the $\mathrm{C}$ compiler.
\end{abstract}

\section{Introduction}

An explicit state model checker is a model checker where all states are explicitly represented in the state space. Explicit model checking is sometimes called stateful state space exploration, especially when checking reachability or safety properties (e.g. deadlocks, assertion violations).

Central to stateful state space exploration is the process of state matching: for every encountered state, it should be checked whether the state has already been visited or not. As the run-time of exploration is linear in the number of transitions (i.e. the amount of newly encountered states and re-visited ones), it is obvious that state matching should be as fast as possible. Typically, hash tables are used to store states. Upon exploration of each state, the hash table is consulted to check whether that state has already been explored or not.

The access to a hash table is through a hash function. Given a key $k$, a hash function $h$ computes the hash code $h(k)$ for this key. This hash code $h(k)$ corresponds to the address in the hash table, where this key $k$ should be stored. For model checking, this $k$ is typically the (binary) representation of a state, called the state vector. Most traditional hash functions compute $h(k)$ by considering all elements of $k$. For example, if $k$ is a string, a typical hash function $h$ would compute $h(k)$ on the basis of all individual characters of $k$. 
With respect to state space exploration, two observations can be made. Firstly, the size of a state vector is usually substantial. State vectors of several hundreds of bytes are not exceptions. This means that computing a traditional hash code for such states can become quite expensive. Secondly, when exploring the state space in a structured manner (e.g. depth first search), the transitions between two consecutive states is local: only a small part of the state changes with respect to the previous state.

This last observation is the idea behind so called incremental hash functions, which use the hash code of a previous key to compute the hash code for the new key. The application of incremental hashing within a model checker is not new. Mehler and Edelkamp [11] have implemented an incremental hashing scheme in the model checker StEAM, a model checker for $\mathrm{C}++$. However, their incremental hashing scheme is only practicable for hashing (large) stacks and queues incrementally. We have improved their hashing scheme by generalising it for hashing vector-based data structures (like state vectors) incrementally by using cyclic polynomials from $[2]$.

Our generalised hashing scheme was originally developed for MoonWALKER $[14]^{3}$ a software model checker for CIL bytecode programs, i.e. .NET applications. Unfortunately, after implementing our incremental hash function into MooNWALKER, initial tests showed no measurable performance gain. We studied this observation using a profiler and found out that the stake of hashing in MooNWALKER is extremely small [12]. Other tasks that have to be performed for each state (e.g. garbage collection, state compression, etc.) take much more time. Any performance gain in hashing would therefore not be visible in the total running time.

The model checker SPIN [6] is arguably one of the fastest explicit state model checkers available. The current version of SpIN uses two traditional hash functions: one composed by Bob Jenkins [20] and one composed by Paul Hsieh [19]. For SPIN verifiers - unlike for bytecode model checkers - hashing accounts for a substantial amount of the running time.

We have implemented our generalised incremental hashing scheme into SPIN 5.1.4. The incremental hashing scheme works for checking safety properties (-DSAFETY) for both 32-bit and 64-bit architectures. Furthermore, it works in exhaustive mode and both approximate modes: bitstate hashing and hash compaction. We have performed numerous experiments on the BEEM benchmarks [16] to compare the incremental hash functions against SPIN's traditional hash functions. From these experiments we learnt that incremental hashing is faster than SPIN's traditional hash implementations without sacrificing too much accuracy. The amount of performance gain depends on several factors:

- the verification mode: exhaustive or approximate,

- the architecture on which the verification is run: 32-bit or 64-bit,

- the size of the state vector, and

\footnotetext{
${ }^{3}$ MoonWalker was previously known as MmC: the Mono Model Checker. Due to several name clashes, MMC has recently been renamed to MoonWALKER.
} 
- the optimisation parameters used for the GCC compiler: the default setting $(-00)$ or the most aggressive optimisation setting (-03), and

- the verifier arguments, e.g., hashtable sizes, maximal search depth.

Incremental hashing performs best for (64-bit) bitstate hashing mode, with larger state vectors and using an optimised verifier.

The rest of the paper is organised as follows. In section 2, we first discuss some related work in the context of (incremental) hashing. Section 3 presents the general incremental hashing scheme: both the intuition of the method and an implementation in $\mathrm{C}$ are discussed in detail. Section 4 explains how we have implemented the method in SPIN and discusses the experimental settings of the benchmark runs that we have conducted. In section 5 we present the results of the benchmark runs and we discuss the outcome of the experiments. Finally, in section 6 we summarise the results and give pointers for future work.

\section{Related Work}

The hash table is the cornerstone of stateful state space exploration. Accesses to a hash table are in amortised $O(1)$ time. Although this is a good worst-case time-complexity, the constant costs are high if a bad hash function is chosen. A good hash function should fulfill the following requirements [10]:

- Fast. The computation of the hash function should be efficient and fast.

- Accurate. To avoid a large number of address collisions, the hash values should distribute evenly over the range of the hash function.

With respect to accuracy, the following rule of thumb is often used: "one bit change in the key should result in half of the bits flipped in its hash code".

A well known hash function for hashing arrays is the rolling hash function [1]. Given a ring $R$, a radix $r \in R$ and a mapping function $T$ that maps array elements to $R$, the rolling hash code for an array $a=a_{0} \ldots a_{n}$ is computed as follows:

$$
H(a)=T\left(a_{0}\right)+r T\left(a_{1}\right)+\ldots+r^{n} T\left(a_{n}\right)=\sum_{i=0}^{n} r^{i} T\left(a_{i}\right)
$$

A possible suitable ring $R$ is $\mathbb{Z} / B$, where $B$ is a prime number and is also the amount of buckets in the hash table. This specialisation of the rolling hash function is called hashing by prime integer division [2].

It is not difficult to see that the rolling hash function is prone to overflow, especially due to the power operations with the radix. Remedying overflow is costly. A recursive formulation of the rolling hash code is less prone to overflow:

$$
\begin{aligned}
H\left(a_{0}\right) & =T\left(a_{0}\right) & \\
H\left(a_{i}\right) & =r H\left(a_{i-1}\right)+T\left(a_{i}\right) & 1 \leq i \leq n
\end{aligned}
$$

Note that the radixes are reversely mapped to the array elements when compared to equation 1, and therefore hash codes derived from the recursive formulation should not be matched against hash codes derived from the non-recursive formulation. 


\subsection{Incremental Hashing}

Karp and Rabin [8] describe an incremental recursive hash function for fast string pattern matching by using recursive hashing by prime integer division. Their idea is to reuse the hash code of the previous unmatched substring for the calculation of the shifted substring. In [2], this is generalised for matching of n-grams.

The rolling hash function is not only amenable for incremental recursive hashing, but also incremental linear hashing. The idea behind incremental linear hashing, is that the contribution of an array element is independent of the contributions of other array elements. In case of an array change, the influence of the old array element is known and thus can be removed, followed by adding the influence of the new array element [2]. In [11], this is expressed as follows. Consider an array $k=v_{0} \ldots v_{i} \ldots v_{n}$ and its successor $k^{\prime}=v_{0} \ldots v_{i}^{\prime} \ldots v_{n}$, then the hash code of $k^{\prime}$ can be computed as follows:

$$
H\left(k^{\prime}\right)=H(k)-r^{i} T\left(k_{i}\right)+r^{i} T\left(k_{i}^{\prime}\right)
$$

Depending on the ring chosen, the power operation with a large index $i$ can easily lead to overflow. Thus using this hashing scheme for arbitrary modification of large arrays is impractical. For stacks and queues however, [11] describes a rewritten version of that formula for push and pop operations with the power operation removed. They tested it in their StEAM model checker, and got at least a speedup factor by 10 compared to non-incremental hashing. Note that this speedup was achieved with fixed-sized stacks of eight megabyte. It is logical to assume that the speedup factor will be much lower with with arbitrary sized stacks.

\subsection{SPIN}

SPIN $[21,6]$ is a state-of-the-art explicit state model checker, which is used as a reference for other model checkers. With respect to memory efficiency and verification time, SpIN is hard to beat. SPIN provides many ways to tune and optimize its verification runs. Most of these optimisation features can be enabled via compilation flags for the C compiler (e.g. GCC). See for details chapter 18 of $[6]{ }^{4}$

SPIN supports three verification modes. The most commonly used verification mode is the exhaustive mode, where all states are stored until the memory to store the states is exhausted. SPIN provides state compression techniques to fit more states in the same amount of memory.

For the cases where there is not enough memory to store all (compressed) states, SpIN supports two lossy, approximate verification modes, which are both heavily based on hashing functions: bitstate hashing and hash compaction. A good survey and extensive discussion on various approximate methods can be found in [10].

\footnotetext{
${ }^{4}$ As this paper is concerned with tuning and optimizing SPIN verification runs, we use several of these compilation parameters, usually prefixed with -D.
} 
Bitstate hashing. Holzmann's bitstate (sometimes called supertrace) hashing [4, 5] algorithm works as follows. Under the assumption that the number of slots in the hash table is high in comparison to the number of reachable states, then it is possible to identify a state by its address in the hash table. In this case, a single bit suffices to indicate whether a state has already been visited or not.

The coverage of bitstate hashing can be improved (on the expense of time) by using $k$ different, independent hash functions for the same hash table. A state is considered visited before if the bits for all $k$ hash functions are set. This variant of bitstate hashing is called $k$-fold bitstate hashing. Triple hashing [3] improves upon this scheme by using three hash values to generate $k$ hashes. For bitstate hashing to be effective, the accuracy of the hash function(s) involved should be high.

Initially, SPIN used $k=2$ by default, but since October 2004, SPIN's default is set to $k=3$. Of course, the variable $k$ can be set to larger values using a run-time option.

Bitstate hashing in SPIN is enabled using the -DBITSTATE parameter.

Hash compaction. Wolper and Leroy [15] introduced hash compaction, a indirection variant of bitstate hashing. The idea of hash compaction is to store the addresses of the occupied bit positions in the bitstate table, rather than storing the whole array itself.

For hash compaction, the hash table is taken to be very large (e.g. $2^{64}$ bits), much too large to fit in memory. Now the address computed by the hash function (e.g. 64 bit-wide) is stored as being a normal state. Hash compaction is thus also viewed as a lossy form of state compression. Hash compaction is more accurate than $k$-fold bitstate hashing (for small $k$ ).

Hash compaction in SPIN is enabled using the -DHC parameter.

The current versions SPIN uses two traditional, linear hash functions:

- Jenkins. Since long, SpIN uses Jenkins hash function [20,7] for both its exhaustive and approximate verification runs. Jenkins' hash function is considered a fast but still quite accurate hash function.

For bitstate hashing, SPIN uses 96-bit and 192-bit versions of Jenkins' hash function. For exhaustive verification, a part of the 96-bit or 192-bit hash value is used.

- Hsieh. Since version 5.1.1 (Nov 2007), SPIN has adopted an alternative hash function by Hsieh [19]. Although perhaps not as accurate as Jenkins, Hsieh's hash function can in some cases be much faster.

Hsieh's hash function can be enabled with the parameter -DSFH, which stands for 'Super Fast Hash'. But Hsieh's hash function is also automatically selected for 32-bit safety runs (-DSAFETY). To speed up such verification runs even further, SPIN's default mask-compression of states is disabled for -DSAFETY runs as well. Hsieh's hash function is not only fast, but its implementation is also suitable for aggressive optimisation by the GCC compiler (i.e. using -02 or -03$)$. 
Currently, there only exist a 32-bit version of Hsieh's hash function. Furthermore, in the current version of SPIN, Hsieh's hash function can only be enabled for checking safety properties.

\section{Generalised Incremental Hashing Scheme}

This section presents the intuition behind the incremental property, the timecomplexity of the incremental hashing scheme and implementation variants. A few concepts, like polynomial rings, from field theory are used to express this. Readers unfamiliar with this may consult [2, Appendix A].

\subsection{Incremental Property}

Consider a Galois field (also known as a finite field) $R=G F(2)[x] /\left(x^{w}+1\right)$, the ring consisting of polynomials in $x$ whose coefficients are 0 or 1 , reduced modulo the polynomial $x^{w}+1$. Make sure that $w$ matches the computer's word size, thus 32 for 32-bits words. The polynomials are represented by $w$-sized bitmasks by placing the coefficients of $x^{i}$ at the $i^{\text {th }}$ bit, creating an one-on-one correspondence between polynomials in $R$ and the bitmasks.

As a radix, the polynomial $x^{\delta} \in R$ is chosen. By setting radix $r=x^{\delta}$, the following incremental hash function is derived from equation 4 :

$$
H\left(k^{\prime}\right)=H(k)+x^{\delta i} T\left(k_{i}\right)+x^{\delta i} T\left(k_{i}^{\prime}\right)
$$

The minus operation from equation 4 is replaced by an + , because addition and subtraction are the same in ring $R$. Now, consider an arbitrary member $q \in R$ with $q(x)=q_{w-1} x^{w-1}+q_{w-2} x^{w-2}+\ldots+q_{0}$. The multiplication of the $x$ and $q(x)$ is the following:

$$
\begin{aligned}
x q(x) & =q_{w-1} x^{w}+q_{w-2} x^{w-1}+\ldots+q_{0} x \\
& =q_{w-2} x^{w-1}+q_{w-3} x^{w-2}+\ldots+q_{0} x+q_{w-1}
\end{aligned}
$$

Equation 7 is equation 6 reduced to modulo $x^{w}+1$. The multiplication by polynomial $x$ results to a left rotate of the coefficients in $q(x)$, hence the name cyclic polynomials. For most platforms, this is an efficient operation. At least all $\mathrm{x} 86$-architectures include native bit rotate instructions. Additions in equation 5 can be implemented using a exclusive-or operation, which is available on all processor platforms.

In order to reduce the amount of operations, equation 5 can be rewritten by applying the associativeness of the + operation, as shown in the next equation:

$$
H\left(k^{\prime}\right)=H(k)+x^{\delta i}\left(T\left(k_{i}\right)+T\left(k_{i}^{\prime}\right)\right)
$$

So far, only one variable is left unmentioned, namely $\delta$. The choice of a $\delta$ for the radix $x^{\delta}$ was experimentally evaluated by [2]. No $\delta$ clearly stood out. For $\delta=1$, the incremental hashing function worked well and they used is subsequently for their experiments. For this reason, we also take 1 for $\delta$. 
Furthermore, as described in [2], cyclic polynomials have one weakness. They have a cycle length of size $w$ for which it computes the hashcode of zero. For example, if a key of size $2 w$ starts with $w$ elements followed by another identical sequence of $w$ elements, then the hashcode for that key is zero. In practise, such keys are extremely rare in model checking, as their size must be exactly $n w$-sized, where $n \in \mathbb{N}$, and that its contents should be also $w$-cyclic as well.

\subsection{Time-complexity}

The time-complexity of the incremental hashing scheme is differently defined compared to traditional hash functions. A fast traditional hash function has a time-complexity in $O(N)$, where $N$ is the array length. The incremental hash function hash function has a time-complexity of $O(1)$ for one change to the array. Theoretically, the incremental hash function is faster if the amount of changes between successive states is smaller than $N$. This is usually the case in model checking, where the amount of changes is usually 1 or 2 and almost never near $N$.

\subsection{Variants}

From the perspective of implementation, there are several variants of the incremental hashing scheme at one's disposal, namely by reordering the coefficients with respect to the bitmask and by using different mappings of function $T$ in equation 4 .

Reordering the Bitmask. The coefficients of polynomials in $R$ were initially mapped to a bitmask whose position coincide with those in the bitmask. This mapping was chosen to allow efficient left-rotate operations on bitmasks as the equivalent to multiplication by $x$. Another ordering of coefficients that works equally well is by ordering the coefficients reversely: the coefficient of $x^{j}$ is placed at the $64-j$ bit position. Such a mapping results to right-rotate operations as the equivalent to multiplication by $x$.

Different Mappings. Our initial experiments with the incremental hashing scheme led to high collision rates. This was caused by the initial mapping of function $T$ in equation 5 , for which we originally chose the identity function. The source of the collisions lied in the entropy of changes between state vectors of successive states. Transitions are often of low entropy, like changing changing a variable from 0 to 1 or add 1 upon variable $i$. The incremental hash function recalculates the hash function upon such changes, but since the entropy is low, the resulting hash would not differ much as one desires for a good hash function. In order to increase entropy, we experimented with different functions of $T$.

Our approach is by using integer hash functions as a $T$. We initially used Wang's integer hash [22], but its constant time-complexity is relatively big compared to that of the incremental hashing scheme, and we observed in experiments that the slowdown made incremental hashing slower than traditional hashing functions. 
The function $T$ has therefore be very fast. An integer hash function for which we observed that it works out well is Knuth's multiplicative constant [9]. This hash function simply multiplies the input by a word-size dependent constant. For 32-bit words, the constant is 2654435769 , and for 64-bits words, the constant is 11400714819323198485 . The constant is calculated by multiplying one-bitmask (i.e., the largest number in the sized word) by the golden ratio $(\sqrt{5}-1) / 2) \approx$ 0.618034 . In [9], Knuth shows this integer hash function has a high likelyhood of spreading the bits through the word, thereby increasing entropy.

Other constants are also applicable. We also experimented with the magic constants of the FNV hash funnction [18], which is 2166136261 for 32-bits words and 14695981039346656037 for 64 -bits words. These FNV constants are 'magic', because their effectiveness was only evaluated by emperic evidence.

\subsection{Implementation Examples}

Here we present several C implementations of the incremental hashing scheme. The implementation below is one for SPIN:

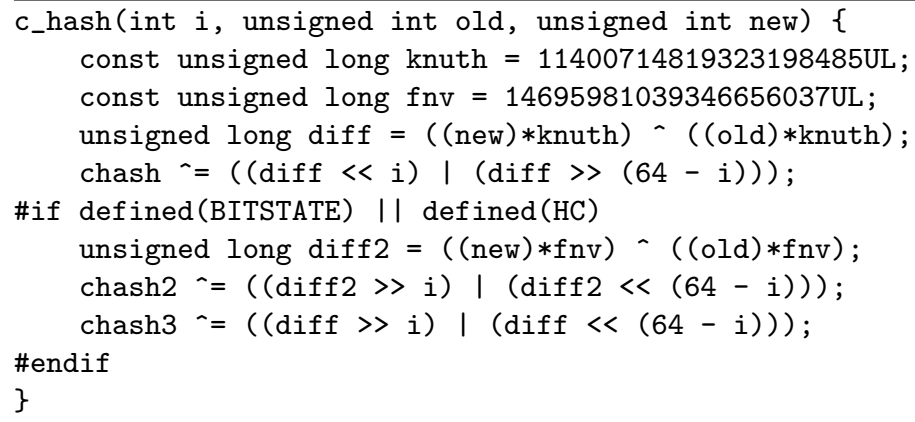

For exhaustive search, only Knuth's multiplicative constant with left-rotatable bitmask are used. For hash compaction, a second hash value is maintained using FNV's constant. For bitstate hashing, triple hashing is used by maintaining a third hash value using a right-rotable bitmask in combination with Knuth's multiplicative constant. We will refer to this implementation as CHASH (where the 'C' stands for cyclic).

Another triple incremental hashing approach is by viewing three words as one word, upon which a bit rotate is performed. This approach is less optimisable because no processor supports a native bit rotate operation for triple-word sized values. We experimented shortly with this approach but found out it always outperformed by the above variant.

\section{Experimental Method}

We originally implemented CHASH in SPIN 4.3.0, and the results with it are described [12]. Since that thesis and this paper, version SpIN 5.1.4 came out and we ported CHASH to it. We subsequently used this newer implementation and benchmarked it extensively against SpIN's default hash functions. 


\subsection{Implementation}

The difficulty of implementing CHASH varies from language to language. In MoONWALKER, which is written in C\#, the implementation was extremely easy due to object encapsulation of the state vector, and therefore also all writes calls to it.

SPIN however is implemented in $\mathrm{C}$ and therefore lacks the expressive means for encapsulated state vector access. The state vector in SPIN is accessible via the global point now and is updated by writing to an offset from this pointer. In order to detect all these writes, which can happen throughout the generated verifier, we had to add a call to the incremental hashing function just before the state vector is updated at that point.

Besides this, we had to overcome several other issues due to specifics in the $\mathrm{C}$ language. For one, our implementation uses the memory address of the written variable as the index argument to function c_hash. This however did not work for the Promela datatypes unsigned int and bit. SpIN uses bitfields as the underlying $\mathrm{C}$ datatype, and bitfields have by definition no addresses. To solve this, we created a virtual memory mapping for unsigned ints and bits. When the verifier is generated, the address of the variable in the symboltable is used as the index instead. We could not use a virtual mapping for all variables because of arrays. Accesses to arrays in SPIN may have an expression as indexer and its value is only known at runtime, not when the verifier is generated.

Also, we could not just use the memory addresses, but we had to use memory offsets. Using offsets is important for approximative methods, because the approximation of the explored state space can slightly differ due to changed memory addresses. These are suspectible to operating system semantics. Offsets are relative and remain the same between runs.

Additionally, we optimised the time-complexity at a small cost of memory. When the DFS search backtracks, CHASH has to be called for a reverse operation in order to calculate the correct corresponding hash code. However instead, we store the hash values on the DFS stack, and write this value as the corresponding hashcode.

\subsection{BEEM Benchmarks}

We used the BEEM benchmark suite for evaluating the effectiveness of the incremental hashing scheme. This suite consists of 57 models, ranging from communication protocols, mutual exclusion algorithms, election algorithms, planning and scheduling solvers and puzzles $[13,16]$. The model are parameterised to yield different problem instances. The total amount of models is 298 and 231 of them are in Promela. We initially evaluated all the Promela models for our experiments. From this evaluation, we made a selection of the 40 largest models that did not run out of memory. These were subsequently used for comparing the different hashing configurations.

Due to space constraints, we are only able to present a selection of the results from these 40 models. We chose to present the ten best problem instances, the 
ten worst problem instances and averages of the forty selected BEEM benchmark suite, thereby giving a nuanced perspective of the results.

\subsection{Setup}

We ran the benchmarks on nine identical nodes, each equipped with Intel Xeon $2.33 \mathrm{GHz}$ processors and $16 \mathrm{~GB}$ memory. When compiling the models, we always enabled the -DSAFETY and -DMEMLIM=15000. As arguments to pan, we fixed the maximal search depth to $20 * 10^{6}$ and disabled stopping on errors and printing unreachable states. Furthermore, we conducted runs with the following compiler flags and pan arguments:

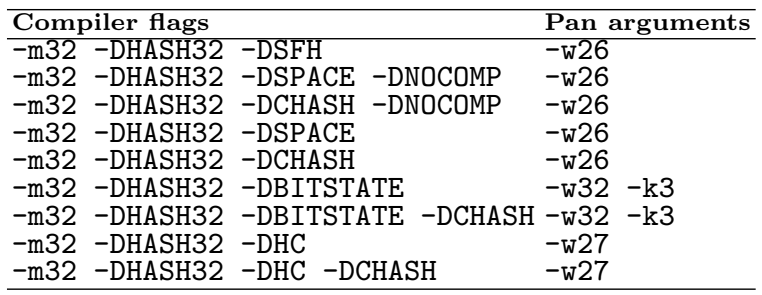

These are 32-bits runs. Additionally, we also ran a series of 64 -bits runs using the following compiler flags and pan arguments:

\begin{tabular}{|c|c|c|}
\hline \multicolumn{2}{|l|}{ Compiler flags } & Pan arguments \\
\hline -m64 -DHASH64 & -DSPACE & $-\mathrm{w} 28$ \\
\hline -m64 -DHASH64 & -DCHASH & $-w 28$ \\
\hline -DHASH64 & -DBITSTATE & - -w36 \\
\hline -DHASH64 & -DBITSTATE -DCHASH & -w36 \\
\hline -DHASH64 & -DHC & -w29 \\
\hline -DHASH64 & -DHC -DCHASH & $-w 29$ \\
\hline
\end{tabular}

All configurations were run twice, namely without compiler optimisations (-00) and with (-03). We furthermore used the GNU profiler on all configurations. All these configurations come down to the total amount 2400 of verifications runs of which we captured their output, processed it and analysed it to present it in the next section.

\section{$5 \quad$ Results and Discussion}

Our benchmark runs generated extensive results which we cannot put all here. We therefore highlight the interesting observations and in case of interest, the full result set is downloadable from the incremental hashing webpage [17].

\subsection{Exhaustive Verification}

In 32-bits exhaustive verification we compared CHASH and Jenkins's against Hsieh's. The result is shown in table 1. The first column is the state vector size, followed by the state space size in $10^{6}$ and transitions in $10^{6}$. Collision rates are indexed against the state space. They can be higher than 100 because Spin 


\begin{tabular}{|c|c|c|c|c|c|c|c|c|c|c|c|c|c|}
\hline \multirow[b]{2}{*}{ model } & \multicolumn{13}{|c|}{ 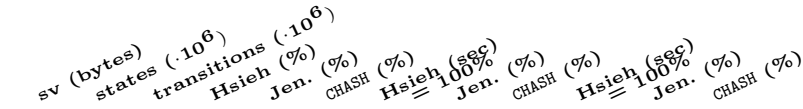 } \\
\hline & & & & & lrat & & & $1 e-O$ & & & $1 \mathrm{e}-\mathrm{OC}$ & & \\
\hline elevator_planning.2 & 48 & 11 & 93 & 939 & 51 & 434 & 47 & 81 & 79 & 54 & 87 & 66 & \\
\hline firewire_link.5 & 404 & 6 & 12 & 3 & 3 & 3 & 24 & 104 & 86 & 34 & 105 & 61 & \\
\hline adding. 6 & 28 & 8 & 12 & 41 & 2 & 2 & 6 & 92 & 87 & 7 & 95 & 78 & \\
\hline telephony.4 & 52 & 12 & 64 & 26 & 20 & 28 & 28 & 99 & 89 & 37 & 106 & 71 & \\
\hline train-gate. 3 & 136 & 20 & 57 & 16 & 16 & 15 & 49 & 101 & 90 & 73 & 102 & 67 & \\
\hline schedule_world.3 & 44 & 4 & 44 & 105 & 21 & 20 & 18 & 101 & 90 & 26 & 102 & 75 & \\
\hline phils.6 & 84 & 14 & 143 & 93 & 92 & 96 & 86 & 108 & 91 & 109 & 103 & 68 & \\
\hline peterson.6 & 44 & 9 & 33 & 13 & 12 & 15 & 16 & 99 & 92 & 21 & 101 & 77 & \\
\hline lann.3 & 128 & 5 & 24 & 8 & 8 & 11 & 24 & 98 & 94 & 33 & 104 & 77 & \\
\hline fischer.6 & 56 & 8 & 33 & 17 & 11 & 11 & 17 & 102 & 94 & 24 & 101 & 76 & \\
\hline$\cdots$ & & & & & & & & & & & & & \\
\hline protocols. 5 & 100 & 3 & 8 & 2 & 2 & 16 & 6 & 106 & 100 & 9 & 101 & 74 & \\
\hline elevator 2.3 & 40 & 8 & 55 & 166 & 22 & 185 & 20 & 105 & 101 & 28 & 99 & 73 & \\
\hline driving_phils. 4 & 84 & 11 & 30 & 6 & 6 & 106 & 15 & 103 & 102 & 22 & 104 & 72 & \\
\hline elevator.3 & 140 & 19 & 70 & 25 & 24 & 37 & 74 & 108 & 103 & 112 & 103 & 79 & \\
\hline lamport_nonatomic.4 & 180 & 16 & 60 & 19 & 19 & 82 & 69 & 98 & 105 & 96 & 101 & 73 & \\
\hline msmie.4 & 100 & 7 & 11 & 2 & 2 & 2 & 10 & 103 & 106 & 16 & 104 & 79 & \\
\hline bridge.2 & 60 & 9 & 27 & 12 & 12 & 12 & 21 & 103 & 107 & 36 & 102 & 89 & \\
\hline sorter.4 & 60 & 13 & 27 & 11 & 8 & 23 & 19 & 102 & 112 & 28 & 103 & 87 & \\
\hline reader_writer.3 & 160 & 1 & 4 & 1 & 1 & 1 & 16 & 99 & 126 & 29 & 101 & 94 & \\
\hline Average & 98 & 12 & 47 & 52 & 17 & 47 & 30 & 102 & 97 & 43 & 102 & 75 & \\
\hline
\end{tabular}

Table 1. 32-bits exhaustive search BEEM benchmark results of Hsieh versus Jenkins (Jen.) and CHASH.

counts each chain hit in the collision chain as a collision. The verification times of Jenkins's and CHASH are indexed against Hsieh's.

The table shows that the average gain of CHASH over Hsieh's is three percent when the models are compiled with -03 and 25 percent when the models are compiled with -00. Later on, we shall discuss the differences between -00 and -03. Most of the ten worst performing models have higher collision rates when used with CHASH in comparison to Jenkins's. It is also noteworthy that Jenkins's has the lowest collision rates (as reflected in the average collision rate), followed by CHASH and Hsieh's.

In 64-bits mode (see table 2) we see that CHASH is on average six percent faster than Jenkins's for -03 and nine percent faster for -00 . This gain is visible on all models for both -00 and -03 , even though the collision rates of CHASH are either on par or worse.

\subsection{Bitstate Hashing}

For bitstate hashing, we denote the accuracy of the search by the coverage indexed against the full state space size, which we know from the exhaustive verifications. Furthermore, we indexed the verification times of CHASH against Jenkins's here.

With 32-bits bitstate hashing (see table 3), we observed with -03 an average performance decrease of two percent using CHASH. With -00 there is a performance gain by 23 percent. With 64 -bits bitstate hashing (see table 4 , we see a performance gain of CHASH by 26 percent (with -03) and 61 percent (with -00).

The coverage rates of Jenkins's shows that it is a good hash function in terms of accuracy. CHASH performs less in that respect, as few models like hanoi.3, protocols.5, sorter.4 and reader_writer.3 have relatively low coverage rates. We 


\begin{tabular}{|c|c|c|c|c|c|c|c|c|c|}
\hline \multicolumn{4}{|l|}{ model } & \multicolumn{2}{|c|}{ collrate } & \multicolumn{2}{|c|}{ time -O3 } & \multicolumn{2}{|c|}{ time -O0 } \\
\hline brp.5 & 148 & 11 & 20 & 1 & 3 & 29 & 61 & 50 & 990 \\
\hline brp.4 & 148 & 7 & 13 & 1 & 4 & 21 & 64 & 35 & 90 \\
\hline driving_phils.4 & 88 & 11 & 30 & 2 & 9 & 22 & 88 & 46 & 83 \\
\hline hanoi.3 & 116 & 14 & 43 & 5 & 7 & 39 & 91 & 91 & 86 \\
\hline phils. 6 & 140 & 14 & 143 & 23 & 23 & 108 & 92 & 243 & 86 \\
\hline elevator2.3_prop4 & 52 & 8 & 55 & 5 & 9 & 26 & 92 & 57 & 88 \\
\hline elevator 2.3 & 52 & 8 & 55 & 5 & 9 & 26 & 92 & 58 & 86 \\
\hline $\operatorname{mcs} .5$ & 68 & 29 & 116 & 10 & 11 & 63 & 93 & 142 & 87 \\
\hline train-gate. 3 & 164 & 20 & 57 & 4 & 29 & 49 & 93 & 123 & 97 \\
\hline telephony.7 & 64 & 22 & 114 & 9 & 15 & 55 & 93 & 130 & 87 \\
\hline schedule_world.3 & 52 & 4 & 44 & 5 & 12 & 23 & 96 & $\mid 52$ & 87 \\
\hline bakery. 5 & 48 & 7 & 25 & 2 & 31 & 13 & 97 & 30 & 82 \\
\hline lann.3 & 140 & 5 & 24 & 2 & 2 & 24 & 97 & 54 & 99 \\
\hline msmie.4 & 180 & 7 & 11 & 0 & 1 & 14 & 97 & 31 & 92 \\
\hline production_cell.4 & 304 & 10 & 42 & 5 & 6 & 50 & 97 & 138 & 97 \\
\hline elevator.3 & 152 & 19 & 70 & 6 & 9 & 74 & 97 & 177 & 93 \\
\hline protocols. 5 & 112 & 3 & 8 & 1 & 7 & 8 & 98 & 17 & 96 \\
\hline frogs. 4 & 68 & 17 & 36 & 2 & 2 & 27 & 98 & 60 & 92 \\
\hline train-gate. 2 & 164 & 18 & 50 & 3 & 30 & 42 & 99 & 108 & 97 \\
\hline reader_writer. 3 & 276 & 1 & 4 & 0 & 1 & 19 & 99 & 39 & 100 \\
\hline Average & 120 & 12 & 47 & 4 & 20 & 34 & 94 & 79 & 91 \\
\hline
\end{tabular}

Table 2. 64-bits exhaustive search BEEM benchmark results of Jenkins (Jen.) versus CHASH

\begin{tabular}{|c|c|c|c|c|c|c|c|c|c|}
\hline \multicolumn{2}{|l|}{ model } & \multicolumn{2}{|c|}{ coverage } & \multirow{2}{*}{\begin{tabular}{|r|}
-O3 \\
18
\end{tabular}} & \multicolumn{2}{|c|}{ rate $-\mathrm{O} 3$} & \multirow{2}{*}{\begin{tabular}{|r|}
$-\mathbf{O 0}$ \\
36
\end{tabular}} & \multicolumn{2}{|c|}{ rate $-\mathrm{OO}$} \\
\hline firewire_link.5 & 6 & 100 & 100 & & 322 & 116 & & 167 & 161 \\
\hline production_cell.4 & 10 & 100 & 100 & 42 & 237 & 109 & \begin{tabular}{|r|} 
\\
2
\end{tabular} & 109 & 153 \\
\hline phils.6 & 14 & 100 & 100 & 86 & 166 & 109 & 134 & 107 & 144 \\
\hline train-gate. 3 & 20 & 100 & 100 & 43 & 465 & 109 & 78 & 253 & 139 \\
\hline train-gate. 2 & 18 & 100 & 100 & 38 & 474 & 108 & 69 & 259 & 137 \\
\hline elevator2.3 & 8 & 100 & 47 & 25 & 302 & 107 & 36 & 211 & 122 \\
\hline fischer.6 & 8 & 100 & 100 & 20 & 426 & 107 & 30 & 280 & 128 \\
\hline elevator_planning.2 & 11 & 100 & 100 & 37 & 311 & 106 & 56 & 204 & 132 \\
\hline telephony.7 & 22 & 100 & 97 & 52 & 419 & 106 & 84 & 262 & 133 \\
\hline brp.5 & 11 & 100 & 100 & 18 & 603 & 105 & 32 & 337 & 135 \\
\hline driving_phils.4 & 11 & 100 & 41 & 17 & 653 & 95 & $|27|$ & $\mid 413$ & 121 \\
\hline elevator.3 & 19 & 100 & 100 & 68 & 274 & 95 & 121 & 154 & 125 \\
\hline $\operatorname{lann} .4$ & 13 & 100 & 99 & 55 & 231 & 92 & 105 & 120 & 123 \\
\hline bridge. 2 & 9 & 100 & 100 & 24 & 392 & 92 & 40 & 230 & 106 \\
\hline msmie.4 & 7 & 100 & 100 & 12 & 605 & 92 & 20 & 359 & 123 \\
\hline frogs. 4 & 17 & 100 & 100 & 26 & 662 & 90 & 42 & 412 & 111 \\
\hline protocols. 5 & 3 & 100 & 63 & 8 & 413 & 89 & 12 & 265 & 111 \\
\hline sorter.4 & 13 & 100 & 89 & 21 & 625 & 89 & 34 & 383 & 117 \\
\hline reader_writer.3 & 1 & 100 & 91 & 16 & 47 & 76 & 30 & 25 & 102 \\
\hline hanoi.3 & 14 & 100 & 0 & 33 & 429 & 4 & 55 & 260 & 6 \\
\hline Average & 12 & 100 & 91 & 31 & 421 & 98 & 51 & 265 & $\overline{123}$ \\
\hline
\end{tabular}

Table 3. 32-bits bitstate search BEEM benchmark results of Jenkins (Jen.) versus CHASH. 
found out this is due to the low entropy input as discussed earlier. Though this is combated using integer hash multiplication, in rare cases as these it is yet insufficient. We found out that additional effective measures are reordering the declaration of variables and/or making the state vector more sparse by adding dummy variables can be quite effective. Depending on the model, we gained nearly on par coverage. A patch against SPIN that generates such models can be downloaded from the incremental hashing webpage. Note that these more unconventional measures are not universally effective and we measured that in general they decrease coverage. For this reason, they are not enabled with CHASH by default.

\begin{tabular}{|c|c|c|c|c|c|c|c|c|c|}
\hline \multicolumn{2}{|l|}{ model } & \multicolumn{2}{|c|}{ coverage } & \multirow{2}{*}{\begin{tabular}{|r|}
$-\mathbf{O 3}$ \\
76 \\
\end{tabular}} & \multirow{2}{*}{\begin{tabular}{|l|} 
rate \\
234
\end{tabular}} & \multirow{2}{*}{$\frac{-\mathrm{O3}}{163}$} & \multirow{2}{*}{$\begin{array}{r}-\mathbf{O 0} \\
138\end{array}$} & \multicolumn{2}{|c|}{ rate $-\mathrm{OO}$} \\
\hline train-gate. 2 & 18 & 100 & 100 & & & & & 129 & 207 \\
\hline production_cell.4 & 10 & 100 & 100 & 77 & 130 & 160 & 158 & 63 & 214 \\
\hline train-gate. 3 & 20 & 100 & 100 & 79 & 250 & 149 & 155 & 128 & 208 \\
\hline production_cell.3 & 6 & 100 & 100 & 67 & 87 & 145 & 131 & 44 & 186 \\
\hline firewire_link.5 & 6 & 100 & 92 & 42 & 141 & 144 & 77 & 77 & 234 \\
\hline phils.6 & 14 & 100 & 100 & 208 & 69 & 137 & 305 & 47 & 196 \\
\hline bakery.7 & 28 & 100 & 100 & 83 & 332 & 136 & 130 & 211 & 161 \\
\hline fischer.6 & 8 & 100 & 100 & 40 & 206 & 135 & 67 & 125 & 170 \\
\hline brp.5 & 11 & 100 & 100 & 38 & 285 & 134 & 65 & 168 & 176 \\
\hline lamport_nonatomic.4 & 16 & 100 & 100 & 111 & 146 & 132 & 179 & 91 & 176 \\
\hline schedule_world. 3 & 4 & 100 & 100 & 38 & 113 & 120 & 60 & 71 & 149 \\
\hline bridge. 2 & 9 & 100 & 100 & 41 & 229 & 120 & 70 & 134 & 133 \\
\hline peterson.6 & 9 & 100 & 100 & 42 & 206 & 120 & 59 & 146 & 141 \\
\hline lamport.7 & 5 & 100 & 100 & 25 & 186 & 119 & 35 & 134 & 140 \\
\hline adding. 6 & 8 & 100 & 100 & 19 & 399 & 115 & 25 & 309 & 129 \\
\hline elevator_planning. 2 & 11 & 100 & 100 & 78 & 147 & 114 & 117 & 98 & 156 \\
\hline at.4 & 7 & 100 & 100 & 32 & 203 & 113 & 49 & 136 & 150 \\
\hline sorter.4 & 13 & 100 & 88 & 39 & 335 & 112 & 64 & 207 & 151 \\
\hline reader_writer.3 & 1 & 100 & 91 & 26 & 29 & 98 & 46 & 16 & 114 \\
\hline protocols.5 & 3 & 100 & 37 & 19 & 165 & 58 & 26 & 119 & 78 \\
\hline Average & 12 & 100 & 97 & 61 & 205 & 126 & 100 & 130 & 161 \\
\hline
\end{tabular}

Table 4. 64-bits bitstate search BEEM benchmark results of Jenkins (Jen.) versus CHASH.

\subsection{Hash-Compaction}

The results from hash-compaction are similar to those from bitstate hashing. Here too we measured a small decline in performance when CHASH is used in 32-bits mode and -03 and a significant performance improvement of 26 percent when -00 is used. See table 5 .

With 64-bits hash-compaction (see table 6), we see that CHASH improves by ten percent over Jenkins's with -03 and 29 percent with -00. For the same reasons as for bitstate hashing, we see that here too a lower coverage goes accompanied by lower performance.

\subsection{Optimisation Flags}

While we ran our benchmarks with and without compiler optimisations enabled, SPIN users usually do without them. XSpin does not enable them by default and 


\begin{tabular}{|c|c|c|c|c|c|c|c|c|c|}
\hline firewire_link.5 & 6 & 100 & 100 & \begin{tabular}{|r|}
-O3 \\
16
\end{tabular} & 372 & 122 & \begin{tabular}{|r|}
$-\mathrm{O} 0$ \\
33
\end{tabular} & 181 & 180 \\
\hline phils.6 & 14 & 100 & 100 & 63 & 229 & 116 & 109 & 132 & 149 \\
\hline train-gate. 2 & 18 & 100 & 100 & 32 & 565 & 110 & 63 & 283 & 140 \\
\hline train-gate. 3 & 20 & 100 & 100 & 36 & 554 & 110 & 71 & 278 & 139 \\
\hline production_cell.4 & 10 & 100 & 100 & 36 & 275 & 109 & 84 & 119 & 158 \\
\hline telephony.7 & 22 & 100 & 97 & 37 & 587 & 109 & 70 & 312 & 137 \\
\hline telephony. 4 & 12 & 100 & 100 & 22 & 566 & 106 & 40 & 304 & 140 \\
\hline lamport_nonatomic. 4 & 16 & 100 & 72 & 45 & 357 & 105 & 91 & 177 & 141 \\
\hline $\operatorname{mcs} .5$ & 29 & 100 & 93 & 42 & 692 & 105 & 72 & 405 & 126 \\
\hline peterson. 6 & 9 & 100 & 100 & 14 & 621 & 105 & 22 & 393 & 123 \\
\hline ‥ 7 & & & & & & & & & \\
\hline lamport.7 & 5 & 100 & 100 & 7 & 661 & 97 & 11 & 423 & 113 \\
\hline driving_phils. 4 & 11 & 100 & 41 & 12 & 923 & 95 & 23 & 489 & 127 \\
\hline protocols.5 & 3 & 100 & 63 & 6 & 561 & 94 & 10 & 327 & 119 \\
\hline lann.4 & 13 & 100 & 99 & 47 & 267 & 93 & 98 & 128 & 126 \\
\hline elevator_planning.2 & 11 & 100 & 100 & 29 & 398 & 91 & 48 & 237 & 121 \\
\hline bridge. 2 & 9 & 100 & 100 & 19 & 497 & 90 & 35 & 264 & 110 \\
\hline msmie. 4 & 7 & 100 & 100 & 9 & 826 & 89 & 16 & 433 & 123 \\
\hline sorter.4 & 13 & 100 & 89 & 16 & 840 & 83 & 29 & 455 & 111 \\
\hline reader_writer.3 & 1 & 100 & 91 & 15 & 50 & 75 & 29 & 26 & 99 \\
\hline hanoi.3 & 14 & 100 & 0 & 25 & 583 & 4 & 47 & 306 & 8 \\
\hline Average & 12 & 100 & 91 & 23 & 582 & 98 & 44 & 322 & 126 \\
\hline
\end{tabular}

Table 5. 32-bits hash-compaction search BEEM benchmark results of Jenkins (Jen.) versus CHASH.

\begin{tabular}{|c|c|c|c|c|c|c|c|c|c|}
\hline \multicolumn{2}{|l|}{ model } & \multicolumn{2}{|c|}{ coverage } & \multirow{2}{*}{\begin{tabular}{|r|}
$-\mathbf{O} 3$ \\
83
\end{tabular}} & \multicolumn{2}{|c|}{ rate $-\mathrm{O} 3$} & \multirow{2}{*}{$\begin{array}{r}-\mathrm{OO} \\
150\end{array}$} & \multicolumn{2}{|c|}{ rate $-\mathrm{OO}$} \\
\hline phils.6 & 14 & 100 & 100 & & 174 & 131 & & 95 & 179 \\
\hline production_cell.4 & 10 & 100 & 100 & 39 & 256 & 128 & 91 & 110 & 150 \\
\hline train-gate. 2 & 18 & 100 & 100 & 36 & 500 & 123 & 73 & 246 & 142 \\
\hline train-gate.3 & 20 & 100 & 100 & 39 & 507 & 122 & 84 & 237 & 149 \\
\hline firewire_link.5 & 6 & 100 & 92 & 19 & 312 & 120 & 38 & 157 & 156 \\
\hline $\operatorname{mcs} .5$ & 29 & 100 & 100 & 54 & 535 & 118 & 94 & 308 & 146 \\
\hline lamport_nonatomic.4 & 16 & 100 & 100 & 49 & 328 & 117 & 98 & 165 & 136 \\
\hline elevator 2.3 & 8 & 100 & 100 & 23 & 332 & 116 & 39 & 199 & 131 \\
\hline telephony.7 & 22 & 100 & 100 & 45 & 493 & 116 & 78 & 281 & 135 \\
\hline production_cell.3 & 6 & 100 & 100 & 34 & 168 & 116 & 78 & 74 & 137 \\
\hline lamport.7 & 5 & 100 & 100 & 12 & 391 & 106 & 17 & 273 & 118 \\
\hline msmie.4 & 7 & 100 & 100 & 13 & 541 & 104 & 23 & 313 & 128 \\
\hline bridge. 2 & 9 & 100 & 100 & 23 & 400 & 104 & 43 & 216 & 107 \\
\hline driving_phils.4 & 11 & 100 & 83 & 16 & 690 & 104 & 27 & 416 & 126 \\
\hline schedule_world.3 & 4 & 100 & 100 & 19 & 220 & 102 & 34 & 126 & 114 \\
\hline adding. 6 & 8 & 100 & 100 & 9 & 836 & 101 & 12 & 634 & 111 \\
\hline sorter.4 & 13 & 100 & 88 & 21 & 628 & 99 & 34 & 388 & 111 \\
\hline elevator_planning.2 & 11 & 100 & 100 & 36 & 317 & 97 & 62 & 185 & 129 \\
\hline reader_writer.3 & 1 & 100 & 91 & 19 & 40 & 94 & 35 & 21 & 101 \\
\hline protocols.5 & 3 & 100 & 37 & 9 & 343 & 51 & 14 & 226 & 68 \\
\hline Average & 12 & 100 & 97 & 29 & 429 & 110 & 53 & 250 & 129 \\
\hline
\end{tabular}

Table 6. 64-bits hash-compaction search BEEM benchmark results of Jenkins (Jen.) versus CHASH. 
users usually forget to enable them manually. Based our results, we see that enabling optimisations (i.e. -03) makes a significant difference, as it reduces the verification time nearly by an half. This substantial improvement costs only a few seconds additional compilation time.

\subsection{Memory Consumption}

We also extracted memory utilisation statistics for runs $^{5}$ with and without CHASH. For both -00 and -03 , we measured an average memory overhead by CHASH of six percent compared to runs with Jenkins's. This is caused by our implementation, which maintains hash values on the DFS stack such that a reverse CHASH operation does not have to be computed.

\subsection{Profiler Runs}

We also wanted to find out how much CHASH improves and whether there is more room for improvement. We ran a profiled version on our selection of the BEEM benchmark suite. For pointing out the interesting points, it sufficies to only present the combined profiler data from 32-bits and 64-bits exhaustive verification. See figures 1 and 2. In this figure, d_hash is Jenkins's hash function, C_hash is the CHASH implementation, hstore is the hashtable storage function, new_state is the DFS routine, compress is the mask-compression function, do_transit performs one transition from the current state and misc are all other functions.

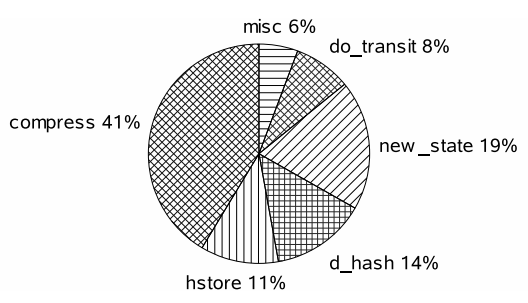

(a) Jenkins.

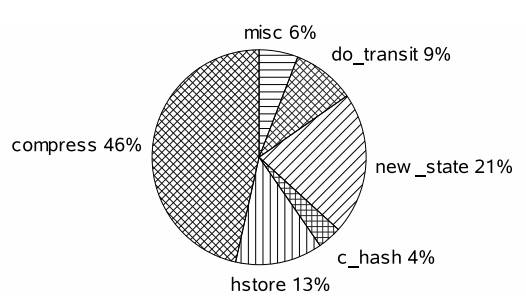

(b) CHASH

Fig. 1. Percentual stakes of five most time-consuming functions for BEEM benchmarks compiled with -00 .

The stake of hashing with Jenkins's is for both -00 and -03 clearly visible in the total running time. When CHASH is enabled, it eliminates hashing as a visible stake in the total running time. For -03, the stake of CHASH is near zero and therefore not depicted in the figure.

Also noteworthy is that compress and hstore have a significant stake for both -00 and -03. The former is in SPIN 5.1.4 disabled by default in case of 32-bit

\footnotetext{
${ }^{5}$ We were not able to include runs with compress disabled for measuring memory overhead, as Spin does not output memory statistics when compress is disabled.
} 


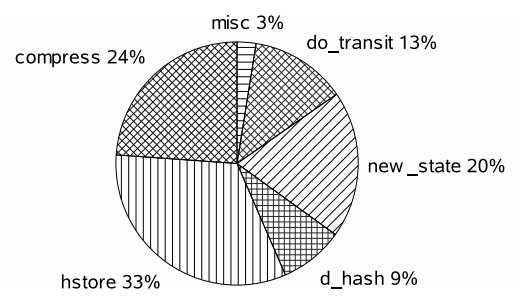

(a) Jenkins.

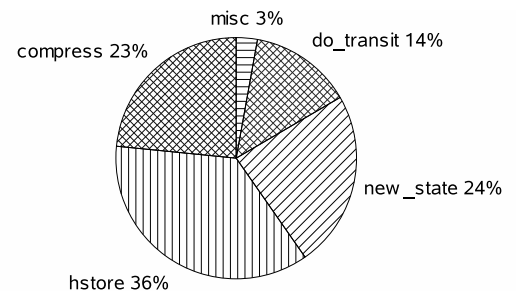

(b) $\mathrm{CHASH}$

Fig. 2. Percentual stakes of five most time-consuming functions for BEEM benchmarks compiled with -03 .

exhaustive verification of safety properties. We measured the impact of this in our benchmarks, and detected that disabling mask-compression improves performance by ten percent in -03 and 36 percent in -00 . This however comes at the cost of increased memory consumption. Unfortunately, this was not measurable because runs with compress disabled do not output memory statistics.

\subsection{Extremely Long Runs}

We also experimented with models that either run out of memory or have a high verification times. We specifically reran the BEEM benchmark with 64-bits bitstate enabled, profiler enabled, compiler optimisations enabled, hashtable size of $2^{36}$, maximal depth of 20 million and $k=3$. The table below is a selection of five models with the longest verification times:

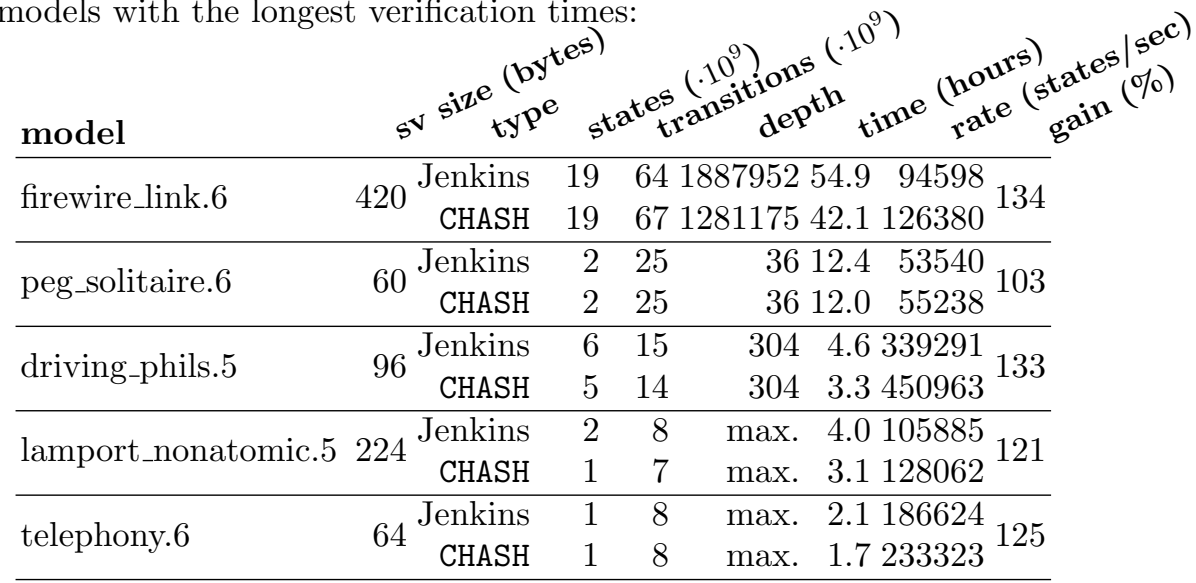

The gain represents the verification rate index of CHASH when compared against Jenkins's. Runs for which the maximal depth was reached are indicated by max. in the depth column.

As can be seen, CHASH improves greatly over Jenkins with an improvement of up to 34 percent. As can be seen from the times, this improvement can save hours of verification. Also particularly interesting is that this selection of models have quite large state vectors. This suggests a correlation between the state vector size and the performance gain by CHASH. The BEEM benchmark suite includes 
too little models with large state vectors and significant large state spaces in order to measure such a correlation.

\section{Conclusions}

There are still several ways to improve our incremental hashing scheme as implemented in Spin. First, we used integer hash functions to improve its uniformity, and though this works out well, there is room for improvement. We saw that for some models, the collision rates were relatively high and/or the coverage rates relatively low. By devising other methods for function $T$, the mapping of integers to the ring $R$, this may be improved. Our experiments with sparse state vectors and variable reordering for bitstate hashing also help, but more investigation is required to define an approach that is on average substantially better.

Also, currently untested is the use of incremental hashing with multi-core model checking (available since Spin 5) and the verification of liveness properties. This is likely to require additions to the CHASH implementation.

CHASH can be also used orthogonally upon traditional hash function, as a good second opinion second hash. This hash code can be stored along the state in the hash table, and used as an additional check before byte-for-byte comparison is done. This can improve the performance of hstore function, of which profiler results have shown that improvements in this function is likely to be reflected in the total running time.

The concept of incremental computation can also be extended to maskcompression and the state collapser. Having an incremental collapse also enables a nicer implementation of incremental hashing, as it will not be necessary anymore to add a c hash at every update of the state vector.

Lastly, the BEEM benchmark suite served their purpose for the greater part of our experiments. It was only lacking on one point, and that is where we wanted to unfold a correlation between the state vector size and the performance gain. The problem lies in the lack of models that have both large state spaces and large state vectors. Adaptations of models in the current suite, or a series of new models that do have those properties would be welcoming for increasing the usefulness of the BEEM benchmark suite even further.

Conclusive, we described an incremental hashing that is applicable to any state vector datastructure, implemented it in SPIN and evaluated it using the BEEM benchmarks. From this evaluation, we observed that SPIN's default settings, namely with compiler optimisations disabled, that incremental hashing is superior to Hsieh's SFH and Jenkins's in all cases. With the most aggressive safe compiler optimisations enabled, namely -03, $\mathrm{SFH}$ is generally better for 32-bits exhaustive search, Jenkins's for all other 32-bits verification modes and incremental hashing is better in all approximate modes and 64-bits in particular. The average reduction of applying compiler optimisation is nearly a half. We recommend it to always enable it, and in case when 64-bits machines are used, combine this with incremental hashing.

The full result set from the BEEM benchmarks and CHASH patch against SPIN 5.1.4 can be downloaded from the incremental hashing webpage [17]. 


\section{References}

1. S. Baase and A. van Gelder. Computer Algorithms. Addison-Wesley, 2000 (3rd).

2. Jonathan D. Cohen. Recursive Hashing Functions for N-grams. Transaction On Information Systems, 15(3):291-320, 1997.

3. Peter C. Dillinger and Panagiotis Manolios. Fast and Accurate Bitstate Verification for SPIN. In Susanne Graf and Laurent Mounier, editors, Model Checking Software - Proc. of the 11th Int. SPIN Workshop (SPIN 2004), Barcelona, April 1-3, 2004, LNCS 2989, pages 57-75. Springer-Verlag, 2004.

4. Gerard J. Holzmann. On Limits and Possibilities of Automated Protocol Analysis. In Proc. 7th Int. Workshop on Protocol Specification, Testing and Verification (PSTV '87), pages 137-161, Amsterdam, 1987. North-Holland.

5. Gerard J. Holzmann. An Analysis of Bitstate Hashing. Formal Methods in System Design, 13:289-307, 1998.

6. Gerard J. Holzmann. The SPIN Model Checker - Primer and Reference Manual. Addison-Wesley, Boston, Massachusetts, USA, 2004.

7. Bob Jenkins. Hash Functions. Dr. Dobbs Journal, 22(9), September 1997.

8. R.M. Karp and M.O. Rabin. Efficient Randomized Pattern-Matching Algorithms. IBM Journal of Research and Development, 31(2):249-260, 1987.

9. Donald E. Knuth. The Art of Computer Programming, Volume 3: (2nd ed.) Sorting and Searching. Addison Wesley Longman Publishing Co., Inc., Redwood City, CA, USA, 1998.

10. Matthias Kuntz and Kai Lampka. Probabilistic Methods in State Space Analysis. In Christel Baier, Boudewijn R. Haverkort, Holger Hermanns, Joost-Pieter Katoen, and Markus Siegle, editors, Validation of Stochastic Systems - A Guide to Current Research, LNCS 2925, pages 339-383. Springer-Verlag, 2004.

11. Tilman Mehler and Stefan Edelkamp. Dynamic Incremental Hashing in Program Model Checking. ENTCS, 149(2):51-69, 2006. Proc. of Third Workshop of Model Checking and Artificial Intelligence (MoChArt 2005).

12. Viet Yen Nguyen. Optimising Techniques for Model Checkers. Master's thesis, University of Twente, Enschede, The Netherlands, December 2007.

13. Radek Pelánek. BEEM: Benchmarks for Explicit Model Checkers. In Dragan Bosnacki and Stefan Edelkamp, editors, Proc. of the 14th Int. SPIN Workshop (SPIN 2007), LNCS 4595, pages 263-267. Springer-Verlag, 2007.

14. Theo C. Ruys and Niels H. M. Aan de Brugh. MMC: the Mono Model Checker. ENTCS, 190(1):149-160, 2007. Proc. of Bytecode 2007, Braga, Portugal.

15. Pierre Wolper and Denis Leroy. Reliable Hashing without Collosion Detection. In Costas Courcoubetis, editor, Proc. of the 5th Int. Conference on Computer Aided Verification (CAV'93), LNCS 697, pages 59-70, 1993.

16. BEEM: BEnchmarks for Explicit Model checkers. http://anna.fi.muni.cz/models/.

17. CHASH - Incremental Hashing for SPIN. http://www-i2.cs.rwth-aachen. de/ nguyen/incrementalHashing.

18. Fowler/Noll/Vo (FNV) Hash. http://isthe.com/chongo/tech/comp/fnv/.

19. Paul Hsieh. Hash functions. http://www.azillionmonkeys.com/qed/hash.html.

20. Bob Jenkins. A Hash Function for Hash Table Lookup. http://burtleburtle.net/bob/hash/doobs.html.

21. SPIN: on-the-fly, LTL model checking. http://spinroot.com/.

22. Thomas Wang. Integer Hash Function. http://www.cris.com/ Ttwang/tech/inthash.htm, 2007. 